\title{
High-frequency ultrasound deformation imaging for adult zebrafish during heart regeneration
}

\author{
Chen Ho-Chiang ${ }^{1}, H_{s i n}$ Huang $^{1}$, Chih-Chung Huang ${ }^{1,2}$ \\ ${ }^{1}$ Department of Biomedical Engineering, ${ }^{2}$ Medical Device Innovation Center, National Cheng Kung University, Tainan, Taiwan \\ Correspondence to: Chih-Chung Huang, PhD. Department of Biomedical Engineering, National Cheng Kung University, 701, No.1, University Rd., \\ Tainan City 70101, Taiwan. Email: cchuang@mail.ncku.edu.tw.
}

\begin{abstract}
Background: The adult human heart cannot efficiently generate new cardiac muscle cells in response to injury, and, therefore, cardiac injury results in irreversible damage to cardiac functions. The zebrafish (Danio rerio) is a crucial animal model in cardiac research because of its remarkable capacity for tissue regeneration. An adult zebrafish can completely regenerate cardiac tissue without a scar being formed, even after $20 \%$ of its ventricular myocardium has been resected. Zebrafish have been utilized in developmental biology and genetics research; however, the details of myocardium motions during their cardiac cycle in different regeneration phases are still not fully understood.
\end{abstract}

Methods: In this study, we used a 70-MHz high-resolution ultrasound deformation imaging system to observe the functional recovery of zebrafish hearts after amputation of the ventricular apex.

Results: The myocardial deformation and cardiac output (CO) were measured in different regeneration phases relative to the day of amputation. In response to the damage to the heart, the peak systolic strain $\left(\varepsilon_{\max }\right)$ and strain during ejection time $\left(\varepsilon_{\mathrm{ej}}\right)$ were lower than normal at 3 days after the myocardium amputation. The CO had normalized to the baseline values at 7 days after surgery.

Conclusions: Our results confirm that the imaging system constructed for this study is suitable for examining zebrafish cardiac functions during heart regeneration.

Keywords: High frequency ultrasound; deformation imaging; zebrafish; heart regeneration

Submitted Jun 28, 2019. Accepted for publication Sep 24, 2019.

doi: 10.21037/qims.2019.09.20

View this article at: http://dx.doi.org/10.21037/qims.2019.09.20

\section{Introduction}

The human heart cannot efficiently generate new cardiac muscle cells in response to injury; therefore, cardiac injury may result in irreversible damage to cardiac functions $(1,2)$. Significant loss of cardiomyocytes during injury and disease can result in heart failure and death. However, nonmammalian vertebrates, such as zebrafish (Danio rerio), exhibit robust regenerative capacity in a variety of tissues, such as the caudal fin, spinal cord, retina, and even—during their larval stages-brain (3-5). In 2002, Poss et al. discovered that adult zebrafish possess the power to regenerate damaged heart muscle (6). When the apex of the zebrafish ventricle is amputated, a large fibrin clot of erythrocytes forms at the wound site and stems bleeding within seconds. Over the following few months, new cardiac tissue is produced and replaces the fibrin clot without a scar being formed (7). An adult zebrafish can completely regenerate its cardiac tissue, even after $20 \%$ ventricular myocardium resection. This regenerative capacity has prompted scientists to investigate the cardiovascular function of zebrafish and its implications for human cardiac pathology (8). Electrocardiography (ECG) of zebrafish has revealed that despite only having a two-chamber heart and lacking pulmonary vasculature, the zebrafish has atrial and ventricular electrical signals similar to those in humans $(9,10)$. When ECG is performed after injury and regeneration in adult zebrafish, abnormal ECG segments and intervals are observed $(2,11)$. Because abnormal ECG segments have been related to myocardial infarction 
and myocardial ischemia in humans (12), the adult zebrafish has become a crucial small-animal model for studying human pathologies in many fields of preclinical drug research aiming to develop novel therapies for heart failure (13), particularly heart failure related to cardiovascular diseases $(14,15)$.

Studies are beginning to reveal the cellular and molecular mechanisms underlying the tissue regeneration ability of zebrafish, and these mechanisms have exciting implications for treatment of human cardiac diseases $(16,17)$. The histology and toxicological pathology of zebrafish have been revealed in studies, but the methods used in these studies cannot elucidate the zebrafish's dynamic cardiac functions. To evaluate the regenerative phases of the zebrafish heart, scientists must evaluate information acquired and stored in vivo experiments. Due to the small size of the zebrafish and the difficulty of performing studies in an aqueous environment, methods for noninvasively assessing zebrafish cardiac morphology and the dynamic functions of the zebrafish heart during regeneration are still limited. Highfrequency ultrasound imaging has been proposed as a suitable tool for achieving high-resolution imaging of adult zebrafish tissue structures and was used in a previous study to sensitively assess the liver tissue of adult zebrafish (18).

A conventional ultrasound imaging device at $8.5 \mathrm{MHz}$ was used to image adult zebrafish hearts (19). A singleelement ultrasound imaging system based on a $45-75-\mathrm{MHz}$ pulsed-wave Doppler device was developed to enable realtime delineation of the cardiac structures of the adult zebrafish (20). A 30-MHz high-frequency ultrasound array system with duplex imaging was also developed to measure adult zebrafish heart motion and blood flow during heart regeneration (21). To investigate the effects of betaadrenergic therapies on the zebrafish heart after myocardial cryoinjury, an ultrasound array system (frequency range $22-55 \mathrm{MHz}$ ) was used to measure heart functions. The ultrasound array system enabled assessment of global and regional myocardial deformation, revealing segmental motion-related disturbances after cryoinjury. According to this assessment, functional recovery of the cryoinjured zebrafish hearts occurred in three distinct phases (22). However, an array system with a center frequency of up to $50 \mathrm{MHz}$ was previously insufficient to monitor the detailed structures of an adult zebrafish heart during regeneration (20). Therefore, our Lab has constructed an $80-\mathrm{MHz}$ ultra-highframe-rate imaging system based on retrospective Dopplergated technology for studying the cardiac functions of adult zebrafish (23). This apparatus was subsequently used to simultaneously measure blood flow signals and capture tissue Doppler images of an adult zebrafish after $15 \%$ of its ventricle had been surgically amputated. Thereafter, we used the $80-\mathrm{MHz}$ high-resolution ultrasound imaging system to track the functional recovery of the damaged heart for 30 days by recording echocardiographic parameters such as A, $\mathrm{S}, \mathrm{Am}$, and $\mathrm{Sm}$ waves (24).

In addition to tissue Doppler imaging, deformation imaging (strain imaging and strain rate imaging) is a novel echocardiographic method that is used to evaluate human cardiac function by measuring regional or global deformation of the myocardium (25-27). In clinical examinations, deformation imaging has been widely used for detecting acute ischemia and myocardial infarction $(28,29)$. A significant correlation between the strain rate and extent of myocardial scarring and infarction in patients was reported (30). Deformation imaging enables comprehensive assessment of myocardial functions through its capacities to register differences between active and passive movement of myocardial segments, quantify intraventricular dyssynchrony, and show components of myocardial function, such as longitudinal myocardial shortening, which is not otherwise visually assessable. Deformation-imagingfacilitated evaluation of adult zebrafish heart functions after amputation may thus provide new information about the animal's heart regeneration mechanism.

In this study, a real-time high-frequency ultrasound deformation imaging system was constructed to perform strain and strain rate imaging for assessing adult zebrafish cardiac functions during various phases of heart regeneration after ventricular myocardium amputation. This paper offers a description of the construction of the deformation imaging system including its signal-processing algorithm and details the hardware used. Animal experiments were performed with adult $\mathrm{AB}$-line zebrafish in which $15 \%$ of the ventricular myocardium had been surgically removed from the apex. The deformation image parameters and cardiac output (CO) were calculated according to deformation images acquired during myocardium recovery. The data reported herein provide a framework for cardiovascular function analysis of the zebrafish heart and may provide insights into zebrafish cardiovascular function during heart regeneration.

\section{Methods}

\section{Zebrafish experimental arrangement}

Experiments were performed using healthy AB-line adult zebrafish (GENDANIO Ocean Technology, New Taipei 
City, Taiwan). The zebrafish were reared in separate tanks at a density of 10 fish per $10 \mathrm{~L}$ of unchlorinated water; a temperature of $27.5-28.5^{\circ} \mathrm{C}$ was maintained. The zebrafish were anesthetized with a stock of ethyl 3-aminobenzoate methanesulfonate salt (MS-222, Sigma-Aldrich, St. Louis, MO, USA) dissolved in water at $\mathrm{pH} 7.2$ to a concentration of $0.16 \%$, kept at $4{ }^{\circ} \mathrm{C}$. For echocardiography examinations, the zebrafish were anesthetized to stage- 4 anesthesia, defined as the zebrafish turning abdominal side up for more than $5 \mathrm{~s}$ but with the operculum still moving. After we put a fish back into fresh water, the fish slowly regained consciousness and began swimming after $2 \mathrm{~min}$. The age and length of the fish used in this study ( $\mathrm{n}=10)$ were $4-6$ months and $3-4 \mathrm{~cm}$ in length, respectively. Experiments were performed on zebrafish with normal hearts (uninjured) and zebrafish with injured hearts (on days 3, 7, 14, and 30 after amputation).

For the heart amputation operation, a zebrafish was anesthetized by immersion in $4.2 \%$ MS-222 (tricaine methanesulfonate) solution and then transferred onto a moist sponge that had been shaped to hold the fish stably upside down. The posterior medial margin of the heart was visually identified, and straight operating scissors were used to puncture the skin. The scissors were used to make a small incision above the heart through the skin and muscles. The silvery epithelial layer of the hypodermis was gently torn using the tip of the scissors to provide direct access to the ventricle. Gentle pressure was applied to expose the ventricle, and the scissors were used to remove $15 \%$ of the ventricle from the apex. The fish were then removed from the moist sponge and immediately placed into the recovery tank, which was a 0.5 -L system tank filled with anesthetic-free system water. The animal survival rate in this study was $95 \%$.

\section{Ultrasound imaging system}

A 70-MHz ultrasound imaging system, which generated images through mechanical scanning, was constructed for obtaining cardiac images of the zebrafish. The experimental system and hardware used to build it are shown in Figure 1. A single-element transducer (NIH Ultrasonic Transducer Resource Center, University of Southern California, Los Angeles, CA, USA) was driven by a pulser-receiver (5900PR, Panametrics, Waltham, MA, USA) with 200-MHz bandwidth. Backscattered signals were amplified by $40 \mathrm{~dB}$ using the pulser-receiver. The signals were then filtered by a bandpass filter and subsequently digitized using an 8-bit analog-to-digital converter (ADC; PXI 5152; National Instruments, Austin, TX, USA) at a sampling rate of $1 \mathrm{GHz}$.
The transducer was mounted on a one-axis piezoceramic motor (HR8; Nanomotion, Yokneam, Israel) to allow mechanical scanning. The transducer could also be moved in the two orthogonal directions using two axial microstep motors (CM1-C-17L30A; Cool Muscle, Tokyo, Japan). The three-axis motor stage was controlled by a motor controller (DMC-1842; Galil Motion Control, Rocklin, CA, USA). A software program was developed for motor control and backscattered signal acquisition using LabVIEW software (National Instruments). The minimal lateral step distance of each scan A-line was $1 \mu \mathrm{m}$. High-resolution twodimensional (2D) B-mode images of the zebrafish hearts were reconstructed by processing the backscattered signals using grayscale imaging. The B-mode imaging frame rate is 10 fps (frame per second). Data were acquired in real time and postprocessed in Matlab (MathWorks Inc., Natick, MA, USA). Deformation imaging was performed and myocardial parameters were measured for the normal zebrafish hearts (uninjured) and the injured zebrafish hearts. The axial and lateral resolutions of the imaging transducer were 30 and $92 \mu \mathrm{m}$, respectively. The aperture size of the transducer is $2 \mathrm{~mm}$ with a focal length of $4 \mathrm{~mm}$. Since the single element transducer was used in this study, the best imaging depth is around the focal zone of transducer. If the imaging region out of the focal zone, the resolution becomes poor which may influence the zebrafish heart imaging and cardiac parameters calculation results. Therefore, the distance between zebrafish heart and transducer was adjusted to keep the imaging region within the focal zone for all experiments.

\section{Blood flow, tissue velocity, and cardiac deformation measurement}

The measurement locations for blood flow and ventricular myocardium motion were determined by consulting the user interface of the real-time ultrasound imaging system. The transducer was automatically moved to the determined locations. Consecutive backscattered signals were then acquired from the moving tissues at each scan location at a pulse repetition frequency of $20 \mathrm{kHz}$ and were then digitized using the ADC. Ultrasound M-mode imaging was then performed to locate the position (window) of blood flow and the myocardium for off-line velocity estimation. After demodulation processing of the backscattered signals, blood flow velocity and tissue velocity were estimated using the following $2 \mathrm{D}$ autocorrelation velocity estimation algorithm (31): 

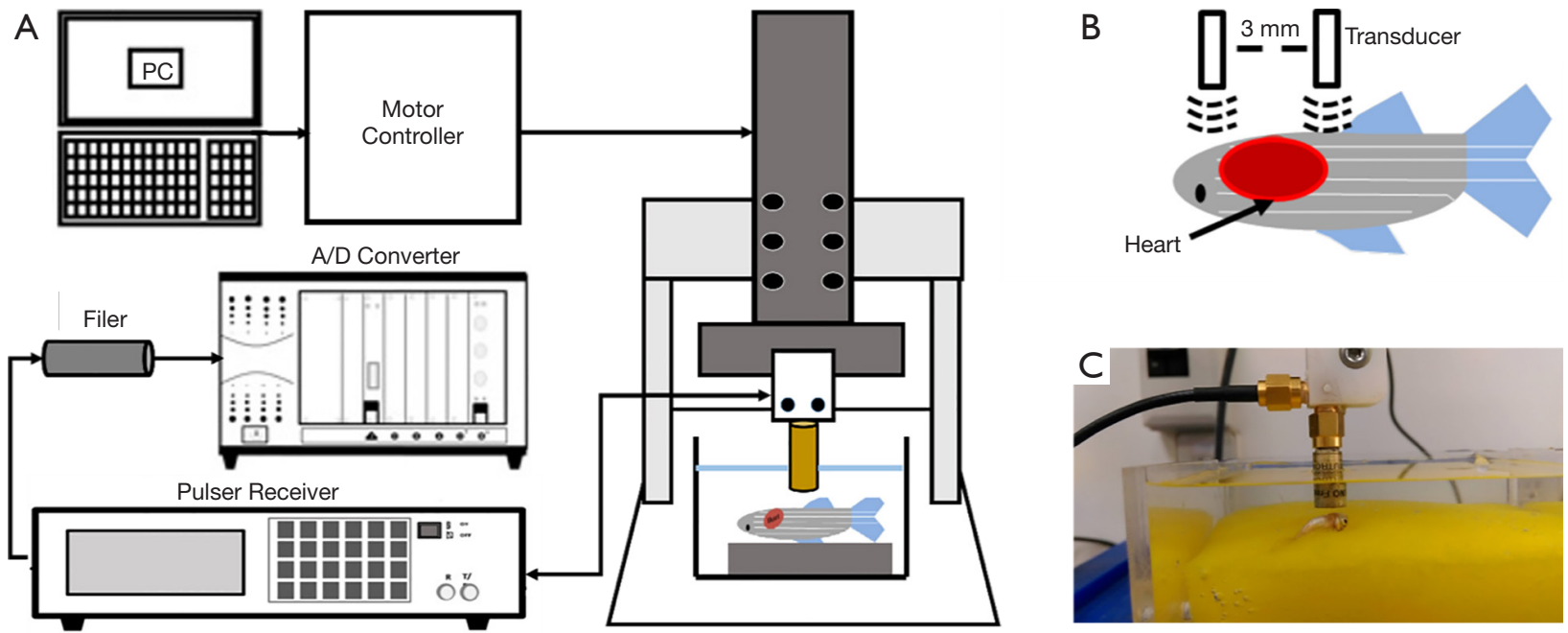

Figure 1 Experimental setup. (A) Block diagram of the experimental system. The location of zebrafish heart imaging (B) and a photo of the experiment (C).

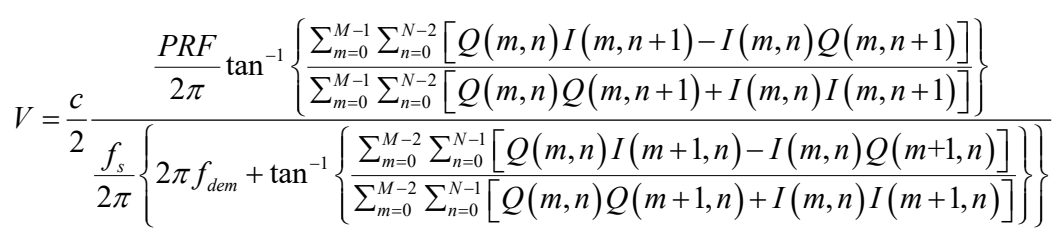

where $\mathrm{V}$ is the estimated velocity; $\mathrm{c}$ is speed of sound in the tissue; $f_{s}$ is the sampling frequency; $f_{\text {dem }}$ is the ratio of the center frequency to the sampling frequency; I and $\mathrm{Q}$ are the in-phase and quadrature-sampled signals, respectively; $M$ is the window depth (quantified as the number of samples); $\mathrm{N}$ is the total number of A-line backscattered signals; and indexes $\mathrm{m}$ and $\mathrm{n}$ correspond to the fast-time (depth direction) and slow-time (pulse number) directions, respectively. In this study, the slow-time window was 100 lines for continuous backscattered signals, and the fast-time window was $30 \mu \mathrm{m}$ (corresponding to the 40 sampling points in the A-line signal). After autocorrelation estimation was conducted, ventricular outflow tract velocity and tissue velocity were obtained. CO is a crucial indicator of the diastolic function of the left ventricle of the heart (32). For acquisition of CO, the diameter $(D)$ of the bulbus arteriosus was determined using $M$-mode imaging. The cross-sectional area of the ventricular outflow tract (VOT area) was also acquired $\left[(D / 2)^{2} \times \pi\right]$. CO was then estimated using the following equation (33):

$$
\mathrm{CO}=\mathrm{VOT} \text { area } \times \text { VOT VTI } \times \text { Heart Rate }
$$

where VOT VTI is the ventricular outflow tract velocity time integral. Figure $2 A$ shows a typical M-mode image of a bulbus arteriosus in a normal zebrafish heart, with a clear distinction between blood flow and tissue (arterial wall) indicated by a speckled pattern. The blood flow velocity (Doppler flow) of the ventricular outflow tract was estimated using the autocorrelation algorithm, and the VOT VTI was measured as illustrated in Figure $2 B$. The $\mathrm{CO}$ was then obtained using equation (2).

In myocardial deformation imaging, the strain rate (SR) can be defined as the change in strain $\left(\Delta " \varepsilon^{\prime \prime}\right)$ per unit of time $(\Delta \mathrm{T})$, which is derived from the formula:

$$
\mathrm{SR}=\frac{\Delta \varepsilon}{\Delta \mathrm{T}}=\frac{\left(\Delta \mathrm{L} / \mathrm{L}_{0}\right)}{\Delta \mathrm{T}}=\frac{\Delta \mathrm{L} / \Delta \mathrm{T}}{\mathrm{L}_{0}}=\frac{\Delta \mathrm{V}}{\mathrm{L}_{0}}
$$

where $\varepsilon$ is the strain, $\mathrm{L}_{\mathrm{O}}$ is the original length, $\Delta \mathrm{L}$ is the change in length, and $\Delta \mathrm{V}$ is the difference in velocities of the myocardium. Integration of SR yields the strain (34):

$$
\varepsilon=\int \mathrm{SR} \cdot \mathrm{dt}
$$

Strain is a measure of tissue deformation and is defined as the change in length divided by the original length. When assessing regional myocardial dysfunction, measured regional strain directly reflects regional function, with positive strain indicating elongation and negative strain 

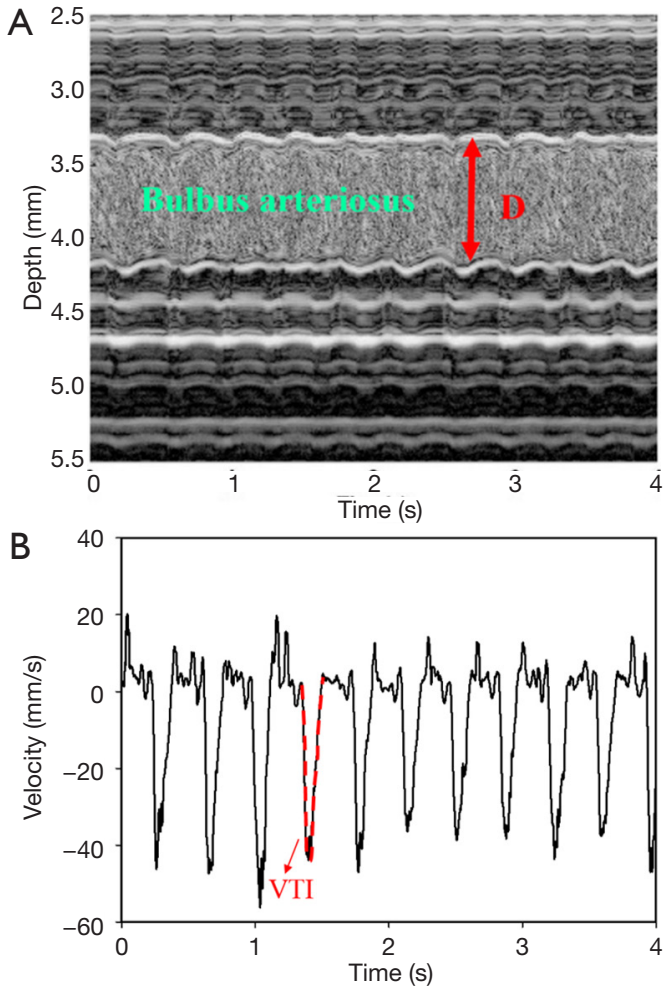

Figure 2 Typical M-mode image of the bulbus arteriosus in a normal zebrafish heart (A) and representative measurement of the ventricular outflow tract velocity time integral (VOT V'TI) (B). D, diameter.

indicating shortening.

\section{Statistical analysis}

The ultrasound deformation imaging parameters were measured in the normal zebrafish hearts (uninjured) and injured zebrafish hearts (on days 3, 7, 14, and 30 after amputation). Differences between the normal and injured groups were assessed using one-way analysis of variance. A probability value of $\mathrm{P}<0.05$ was considered indicative of a statistically significant difference (relative to the normal zebrafish group). Statistical analysis was performed using commercial software (SigmaPlot 12.0; Systat Software, San Jose, CA, USA).

\section{Results}

Typical B-mode and M-mode images of a zebrafish heart acquired by the $70-\mathrm{MHz}$ ultrasound imaging system are displayed in Figure 3A,B, respectively. The B-mode image shows the major cardiac anatomy of a normal zebrafish heart,
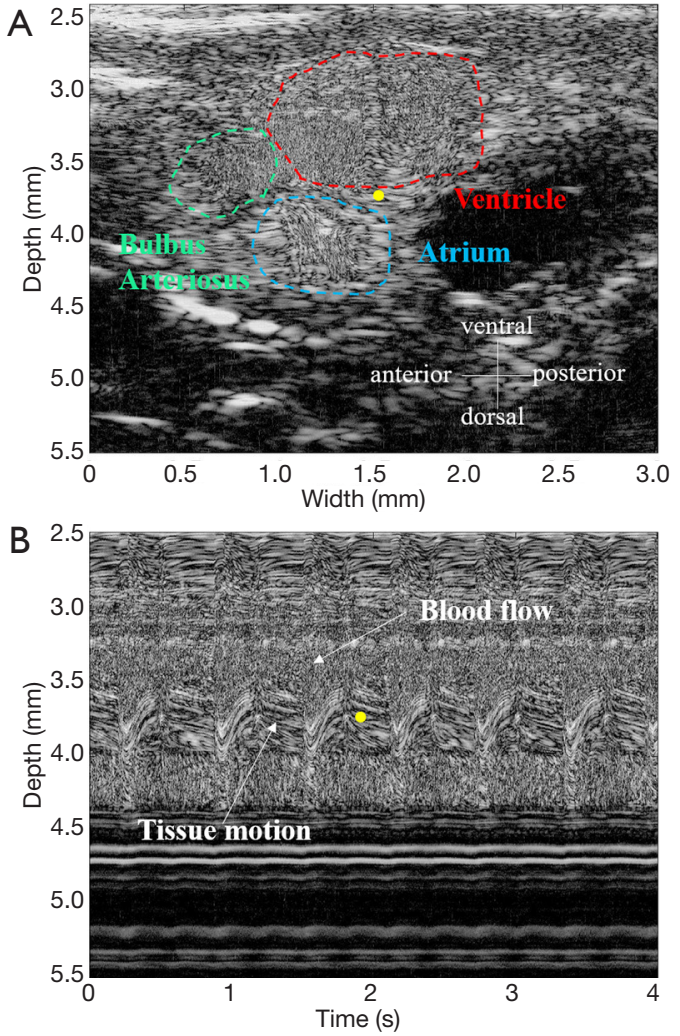

Figure 3 Typical B-mode (A) and M-mode (B) images of a normal zebrafish heart and its corresponding cardiac anatomical positions, obtained using a high-frequency ultrasound imaging system. The yellow dots in the images represent corresponding measurement positions in the heart.

including the ventricle, atrium, and bulbus arteriosus. The blood flow into and out of the ventricle are clearly evident in the image. Blood drains into the triangular single-chambered atrium from the sinus venosus and is then pumped by the single-chambered ventricle through the bulboventricular foramen into the pear-shaped bulbus arteriosus, which is situated between the ventricle and the ventral aorta. Figure 4 shows examples of quantitative measurements of blood flow velocity and myocardium motion velocity during the cardiac cycle of a normal zebrafish heart. This figure combines information on the blood flow pattern in the ventricle and the tissue motion pattern in the atrioventricular valve annulus to show the relationship between these phenomena in the zebrafish heart. Understanding this relationship facilitates evaluation of cardiac functions during different heart regeneration phases.

Figure 5 presents typical strain and strain rate images of an adult zebrafish heart and provides examples of 

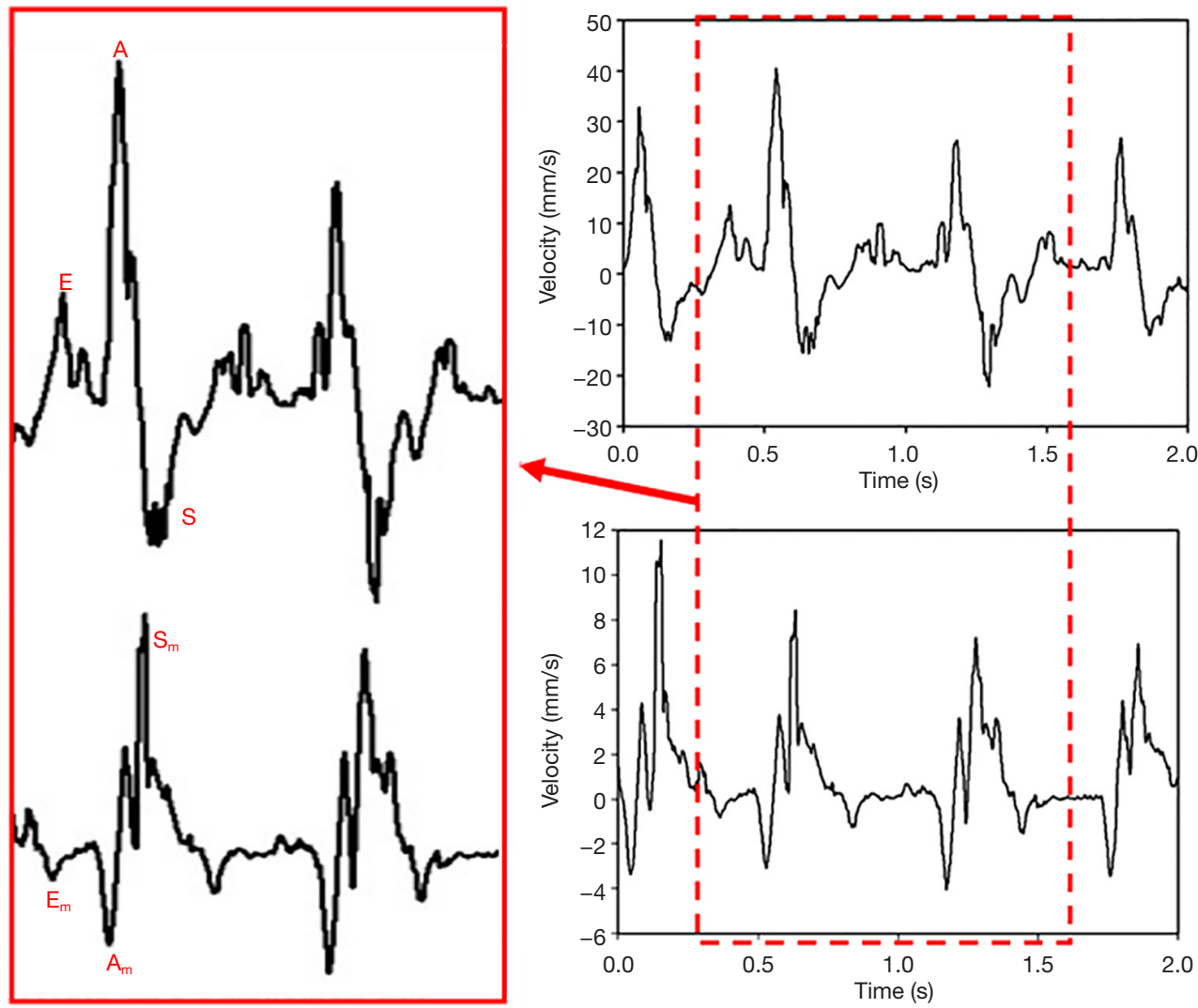

Figure 4 Blood flow velocity (top) and tissue motion velocity (below) waveforms for a normal zebrafish heart. E, early diastolic inflow; A, late diastolic inflow; S, ventricular systolic outflow; Em, early diastolic myocardial motion; Am, late diastolic myocardial motion; Sm, systolic myocardial motion.

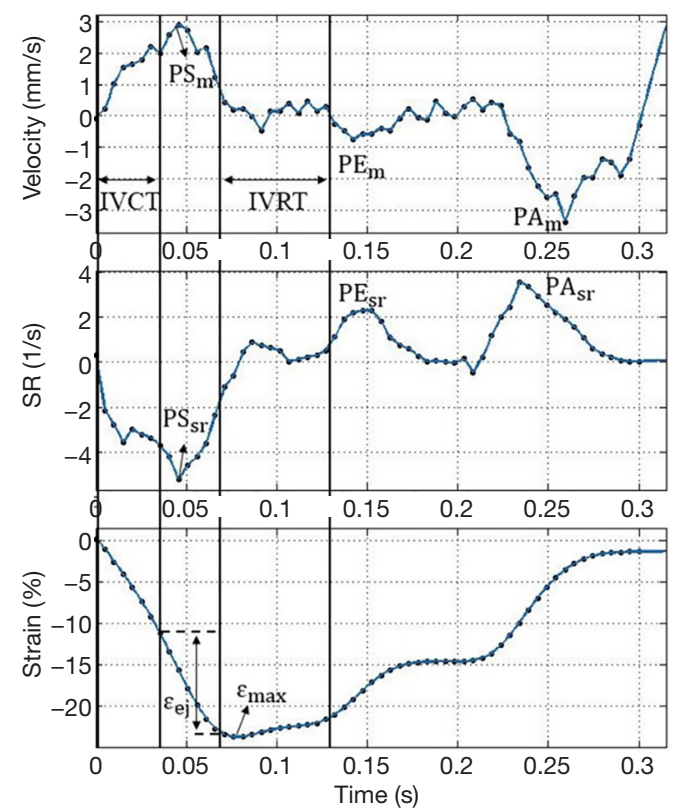

Figure 5 Typical curves of regional tissue velocity, strain, and strain rate for an adult zebrafish during one cardiac cycle. quantitative measurement of myocardial deformation during the cardiac cycle. Several parameters used to characterize myocardium deformation are clearly displayed: isovolumetric contraction time (IVCT), isovolumetric relaxation time (IVRT), peak velocity of systolic myocardial motion $\left(\mathrm{PS}_{\mathrm{m}}\right)$, peak velocity of early diastolic myocardial motion $\left(\mathrm{PE}_{\mathrm{m}}\right)$, peak velocity of late diastolic myocardial motion $\left(\mathrm{PA}_{\mathrm{m}}\right)$, peak strain rate of systolic myocardial motion $\left(\mathrm{PS}_{\text {sr }}\right.$ ), peak strain rate of early diastolic myocardial motion $\left(\mathrm{PE}_{\mathrm{sr}}\right)$, peak strain rate of late diastolic myocardial motion $\left(\mathrm{PA}_{\mathrm{sr}}\right)$, peak systolic strain during the entire heart cycle $\left(\varepsilon_{\max }\right)$, and strain during ejection time $\left(\varepsilon_{\mathrm{e}}\right) . \mathrm{PS}_{\mathrm{sr}}, \mathrm{PE}_{\mathrm{sr}}$, and $\mathrm{PA}_{\mathrm{sr}}$ did not fluctuate during the various heart regeneration phases, whereas $\varepsilon_{\max }$ and the strain $\varepsilon_{\mathrm{ej}}$ were significantly decreased at 3 days after the myocardium amputation $(-14.7 \%$ and $-7.1 \%$, respectively). However, these parameters had returned to the baseline values for a normal heart at 14 days after surgery. The quantitative data of these parameters are listed in Table 1. Figure $6 A, B$ respectively show B-mode images of an adult zebrafish heart with the epidermis 
Table 1 Cardiac parameters of the adult zebrafish during various phases of regeneration

\begin{tabular}{|c|c|c|c|c|c|}
\hline Parameters & Normal & Day 3 & Day 7 & Day 14 & Day 30 \\
\hline IVRT (s) & $0.0957 \pm 0.02$ & $0.0867 \pm 0.014$ & $0.0904 \pm 0.024$ & $0.103 \pm 0.017$ & $0.0938 \pm 0.019$ \\
\hline HR (beats/min) & $118.8 \pm 26.5$ & $143.4 \pm 38.3^{*}$ & $127.8 \pm 34.6$ & $105.8 \pm 29.3$ & $115.8 \pm 31.4$ \\
\hline $\mathrm{PS}_{\mathrm{m}}(\mathrm{mm} / \mathrm{s})$ & $7.71 \pm 2.79$ & $7.33 \pm 1.92$ & $7.59 \pm 2.11$ & $7.84 \pm 1.79$ & $7.64 \pm 2.23$ \\
\hline $\mathrm{PA}_{\mathrm{m}}(\mathrm{mm} / \mathrm{s})$ & $-5.51 \pm 2.03$ & $-6.11 \pm 3.17$ & $-5.82 \pm 2.79$ & $-5.93 \pm 3.03$ & $-5.73 \pm 2.61$ \\
\hline $\mathrm{PS}_{\mathrm{sr}}\left(\mathrm{s}^{-1}\right)$ & $-4.48 \pm 0.94$ & $-4.19 \pm 1.15$ & $-4.24 \pm 1.07$ & $-4.63 \pm 0.87$ & $-4.56 \pm 1.03$ \\
\hline$P E_{s r}\left(s^{-1}\right)$ & $2.1 \pm 1.03$ & $2.87 \pm 1.55$ & $2.47 \pm 1.35$ & $2.33 \pm 1.69$ & $2.41 \pm 1.27$ \\
\hline $\mathrm{PA}_{\mathrm{sr}}\left(\mathrm{s}^{-1}\right)$ & $3.11 \pm 1.54$ & $3.74 \pm 0.93$ & $3.41 \pm 1.31$ & $3.32 \pm 1.74$ & $3.37 \pm 1.46$ \\
\hline$\varepsilon_{\mathrm{ej}}(\%)$ & $-13.3 \pm 2.98$ & $-7.1 \pm 3.46^{\star}$ & $-11.2 \pm 2.22$ & $-14.6 \pm 3.13$ & $-13.8 \pm 2.64$ \\
\hline
\end{tabular}

${ }^{*}, \mathrm{P}<0.05$.
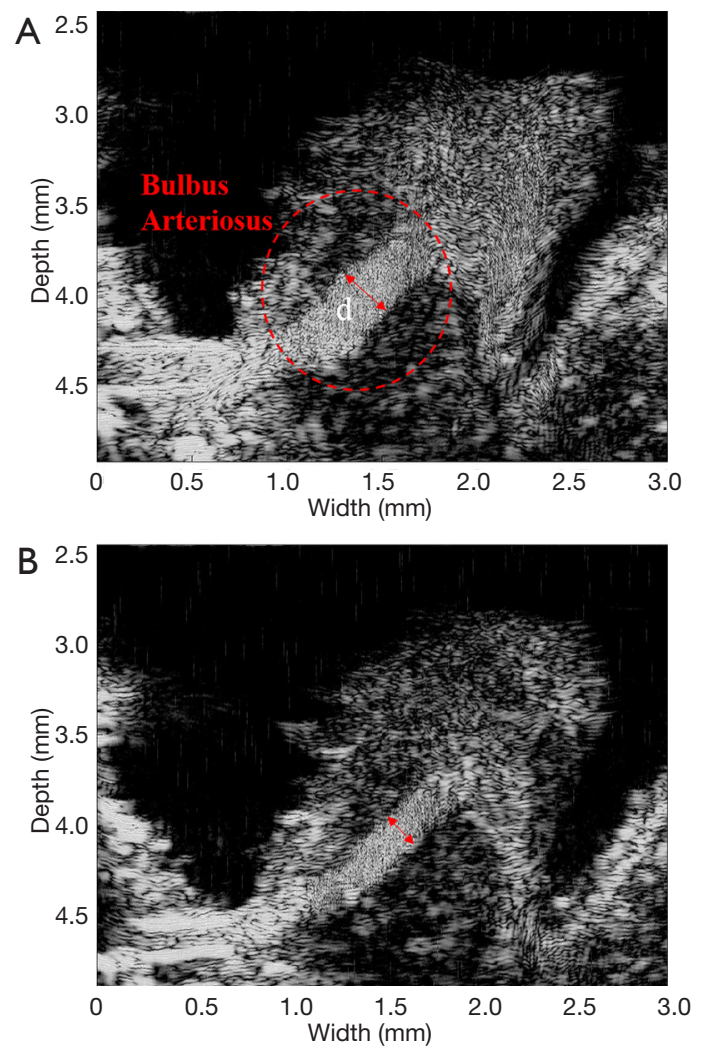

Figure 6 B-mode images of an adult zebrafish heart with epidermis removed prior to $(\mathrm{A})$ and at 1 day postamputation (B). d, diameter. completely removed prior to and at 1-day postamputation. Since the epidermis was removed, the fish heart was exposed directly for ultrasound imaging. There are no surrounding tissues around the heart compared to Figure 3 (without epidermis removed).

\section{Discussion}

Figure $3 B$ shows a typical M-mode image of a normal zebrafish heart and its corresponding measurements of anatomical locations. The atrioventricular valve annulus (dorsal side of the ventricle) was selected as the measurement location for the ventricular myocardium because ventricular motion is greatest at this site. Additionally, the location of the atrioventricular valve annulus corresponds to the human heart's mitral annulus, the velocity of which is often measured using tissue Doppler imaging to assess cardiac functions. The blood flow and tissue motion locations were easily identified by a speckled pattern in the M-mode imaging. The transient information of blood flow velocity and tissue motion velocity within the ventricle were also analyzed.

Several flow parameters for characterizing blood flow velocity are clearly displayed in the Figure 4: the early diastolic inflow (early filling E-wave), late diastolic inflow 
(late filling A-wave), and ventricular systolic outflow (S-wave). The measured waveforms used to characterize the myocardium motion velocity during the various phases of the cardiac cycle are also presented: the early diastolic myocardial motion (Em-wave), late diastolic myocardial motion (Am-wave), and systolic myocardial motion (Smwave). The images clearly show that the tissue motion velocity was much slower than the blood flow velocity. In addition, the Doppler signals of velocities of tissue motion and blood flow have opposite directions, which indicates that tissue motion leads the cardiac phases.

$\mathrm{CO}$ in the adult zebrafish decreased significantly after the amputation. The $\mathrm{CO}$ was $41.26 \pm 4.17 \mu \mathrm{L} / \mathrm{min}$ (mean \pm standard deviation) for the normal zebrafish heart and $15.47 \pm 5.02 \mu \mathrm{L} / \mathrm{min}$ for the injured zebrafish heart. These findings are reasonable because, after injury, the heart must reduce its $\mathrm{CO}$ to maintain circulation and thus keep the fish alive; the heart can then be regenerated gradually through various cellular and molecular mechanisms. Diseases of the cardiovascular system, particularly the pandemic diseases of cardiomyopathy and heart failure, are often associated with changes in $\mathrm{CO}(35,36)$. Cardiac deformation and $\mathrm{CO}$ followed the same trend over the period of regeneration as is described by the equation (37):

$$
\mathrm{CO}=\mathrm{VOT} \text { area } \times \mathrm{VOT} \mathrm{VTI} \times \mathrm{HR}=\mathrm{SV} \times \mathrm{HR}
$$

where SV indicates the stroke volume (volume of blood pumped from the ventricle per beat). $\mathrm{CO}$ can be determined by the SV and is directly related to the cardiac chamber size during the diastole and systole (cardiac deformation). Therefore, the experimental results of $\mathrm{CO}$ and cardiac deformation are consistent in this study.

The diameter of the bulbus arteriosus was smaller after surgery, as shown in Figure 6; this may be another reason why the CO dropped dramatically. Because there were no significant changes in VOT velocity after surgery, the decrease in $\mathrm{CO}$ can be attributed to variation in the VOT area. Thus, the bulbus arteriosus plays an important role in modulating zebrafish cardiac function after heart injury.

In human cardiology, myocardial infarction and arrhythmia result from the formation of scar tissue and irreversible loss of ventricular function. Myocardial scarring, which lacks a contractile phenotype and constitutes a substrate for arrhythmia, occurs during myocardial infarction or as a result of surgical treatment of congenital heart disease. Patients with acute anterior myocardial infarction were examined using longitudinal strain Doppler echocardiography after coronary reperfusion (29). The results showed that global and regional strain both decreased after reperfusion therapy and were correlated with the extent of the myocardial infraction. Human hearts have a limited capacity to regenerate from significant loss of myocardium, and injured hearts heal by forming scar tissues. Unlike that in human hearts, scar tissue in zebrafish may disappear completely after injury. The results of this study demonstrate that the cardiac deformation parameters gradually returned to the baseline values for a healthy zebrafish heart after surgery, which agrees with our expectation of scar disappearance.

\section{Conclusions}

A high-frequency ultrasound deformation imaging (strain imaging and strain rate imaging) methodology was employed to investigate adult zebrafish in this study. Several cardiac parameters of the zebrafish heart were measured to assess dynamic cardiac functions during various phases of heart regeneration. In response to heart damage, the peak systolic strain $\left(\varepsilon_{\max }\right)$ and strain during ejection time $\left(\varepsilon_{\mathrm{e}}\right)$ were lower than normal at 3 days after myocardium amputation. Subsequently these values returned to the baseline for a normal zebrafish heart. CO had also dropped dramatically by the 3-day mark but had gradually normalized to the baseline value at 7 days after surgery. Besides the heart itself, the bulbus arteriosus seemed to play a critical role in modulating cardiac function after heart injury. In most cases of heart disease in humans, cardiac functions cannot be completely recovered even after relatively successful treatment. However, the unique cardiac function of the zebrafish may provide new insights into heart regeneration. Finally, in this study, we demonstrated the usefulness of high-frequency ultrasound deformation imaging for investigating the cardiac functions of small animals in vivo. The technique is simple and relatively inexpensive and may be used for the further study of cardiac function in adult zebrafish.

\section{Acknowledgments}

Funding: This work was supported by National Health Research Institutes in Taiwan under grant NHRI-EX107$10712 \mathrm{EI}$ and the Ministry of Science and Technology of Taiwan under grant MOST 107-2221-E-006-024-MY3, and a part was supported by the Medical Device Innovation Center (MDIC), National Cheng Kung University from the Featured Areas Research Center Program within the framework of the Higher Education Sprout Project by the 
Ministry of Education (MoE) in Taiwan.

\section{Footnote}

Conflicts of Interest: The authors have no conflicts of interest to declare.

\section{References}

1. van den Borne SW, Diez J, Blankesteijn WM, Verjans J, Hofstra L, Narula J. Myocardial remodeling after infarction: the role of myofibroblasts. Nat Rev Cardiol 2010;7:30-7.

2. Chablais F, Veit J, Rainer G, Jaźwińska A. The zebrafish heart regenerates after cryoinjury-induced myocardial infarction. BMC Dev Biol 2011;11:21.

3. Hu N, Sedmera D, Yost HJ, Clark EB. Structure and function of the developing zebrafish heart. Anat Rec 2000;260:148-57.

4. Kizil C, Kaslin J, Kroehne V, Brand M. Adult neurogenesis and brain regeneration in zebrafish. Dev Neurobiol 2012;72:429-61.

5. Unguez GA. Electric fish: new insights into conserved processes of adult tissue regeneration. J Exp Biol 2013;216:2478-86.

6. Poss KD, Wilson LG, Keating MT. Heart regeneration in zebrafish. Science 2002;298:2188-90.

7. Raya A, Koth CM, Büscher D, Kawakami Y, Itoh T, Raya RM, Sternik G, Tsai HJ, Rodríguez-Esteban C, IzpisúaBelmonte JC. Activation of Notch signaling pathway precedes heart regeneration in zebrafish. Proc Natl Acad Sci U S A 2003;100 Suppl 1: 11889-95.

8. Dahme T, Katus HA, Rottbauer W. Fishing for the genetic basis of cardiovascular disease. Dis Model Mech 2009;2:18-22.

9. Milan DJ, Jones IL, Ellinor PT, MacRae CA. In vivo recording of adult zebrafish electrocardiogram and assessment of drug-induced QT prolongation. Am J Physiol Heart Circ Physiol 2006;291:H269-73.

10. Sedmera D, Reckova M, deAlmeida A, Sedmerova M, Biermann M, Volejnik J, Sarre A, Raddatz E, McCarthy RA, Gourdie RG, Thompson RP. Functional and morphological evidence for a ventricular conduction system in zebrafish and Xenopus hearts. Am J Physiol Heart Circ Physiol 2003;284:H1152-60.

11. Sun P, Zhang Y, Yu F, Parks E, Lyman A, Wu Q, Ai L, $\mathrm{Hu}$ CH, Zhou Q, Shung K, Lien CL, Hsiai TK. Microelectrocardiograms to study post-ventricular amputation of zebrafish heart. Ann Biomed Eng 2009;37:890-901.

12. Cimponeriu A, Starmer CF, Bezerianos A. A theoretical analysis of acute ischemia and infarction using ECG reconstruction on a 2-D model of myocardium. IEEE Trans Biomed Eng 2001;48:41-54.

13. Patten RD, Hall-Porter MR. Small animal models of heart failure. Circ Heart Fail 2009;2:138-44.

14. Hill A. Zebrafish in drug discovery: Safety assessment. In: Drug discovery and evaluation: safety and pharmacokinetic assays. Berlin: Springer Berlin Heidelberg; 2013, 605-629.

15. Rocke J, Lees J, Packham I, Chico T. The zebrafish as a novel tool for cardiovascular drug discovery. Recent Pat Cardiovasc Drug Discov 2009;4:1-5.

16. Hecker L, Khait L, Sessions SK, Birla RK. Functional evaluation of isolated zebrafish hearts. Zebrafish 2008;5:319-22.

17. Major RJ, Poss KD. Zebrafish heart regeneration as a model for cardiac tissue repair. Drug Discov Today Dis Models 2007;4:219-25.

18. Goessling W, North TE, Zon LI. Ultrasound biomicroscopy permits in vivo characterization of zebrafish liver tumors. Nat Methods 2007;4:551-3.

19. Ho YL, Shau YW, Tsai HJ, Lin LC, Huang J, Hsieh FJ. Assessment of zebrafish cardiac performance using Doppler echocardiography and power angiography. Ultrasound Med Biol 2002;28:1137-43.

20. Sun L, Lien CL, Xu X, Shung K. In vivo cardiac imaging of adult zebrafish using high frequency ultrasound (45-75 MHz). Ultrasound Med Biol 2008;34:31-9.

21. Kang BJ, Park J, Kim J, Kim HH, Lee C, Hwang JY, Lien CL, Shung KK. High-frequency dual mode pulsed wave Doppler imaging for monitoring the functional regeneration of adult zebrafish hearts. J R Soc Interface 2015;12. doi: 10.1098/rsif.2014.1154.

22. Hein SJ, Lehmann LH, Kossack M, Juergensen L, Fuchs D, Katus HA, Hassel D. Advanced echocardiography in adult zebrafish reveals delayed recovery of heart function after myocardial cryoinjury. PLoS One 2015;10:e0122665.

23. Liu TY, Lee PY, Huang CC, Sun L, Shung KK. A study of the adult zebrafish ventricular function by retrospective Doppler-gated ultrahigh-frame-rate echocardiography. IEEE Trans Ultrason Ferroelectr Freq Control 2013;60:1827-37.

24. Huang CC, Su TH, Shih CC. High-resolution tissue Doppler imaging of the zebrafish heart during its regeneration. Zebrafish 2015;12:48-57.

25. Gorcsan J 3rd, Tanaka H. Echocardiographic assessment of myocardial strain. J Am Coll Cardiol 2011;58:1401-13. 
26. Shah AM, Solomon SD. Myocardial deformation imaging: current status and future directions. Circulation 2012;125:e244-8.

27. Dandel M, Lehmkuhl H, Knosalla C, Suramelashvili N, Hetzer R. Strain and strain rate imaging by echocardiography-basic concepts and clinical applicability. Curr Cardiol Rev 2009;5:133-48.

28. Edvardsen T, Skulstad H, Aakhus S, Urheim S, Ihlen H. Regional myocardial systolic function during acute myocardial ischemia assessed by strain Doppler echocardiography. J Am Coll Cardiol 2001;37:726-30.

29. Jain M, Cui L, Brenner DA, Wang B, Handy DE, Leopold JA, Loscalzo J, Apstein CS, Liao R. Increased myocardial dysfunction after ischemia-reperfusion in mice lacking glucose-6-phosphate dehydrogenase. Circulation 2004;109:898-903.

30. Vartdal T, Brunvand H, Pettersen E, Smith HJ, Lyseggen E, Helle-Valle T, Skulstad H, Ihlen H, Edvardsen T. Early prediction of infarct size by strain Doppler echocardiography after coronary reperfusion. J Am Coll Cardiol 2007;49:1715-21.

31. Loupas T, Peterson RB, Gill RW. Experimental evaluation of velocity and power estimation for ultrasound blood flow imaging, by means of a two-dimensional autocorrelation approach. IEEE transactions on ultrasonics, ferroelectrics, and frequency control. IEEE Trans Ultrason Ferroelectr Freq Control 1995;42:689-99.

Cite this article as: Ho-Chiang C, Huang H, Huang CC. High-frequency ultrasound deformation imaging for adult zebrafish during heart regeneration. Quant Imaging Med Surg 2020;10(1):66-75. doi: 10.21037/qims.2019.09.20
32. Nagueh SF, Appleton CP, Gillebert TC, Marino PN, Oh JK, Smiseth OA, Waggoner AD, Flachskampf FA, Pellikka PA, Evangelisa A. Recommendations for the evaluation of left ventricular diastolic function by echocardiography. Eur J Echocardiogr 2009;10:165-93.

33. Funk DJ, Moretti EW, Gan TJ. Minimally invasive cardiac output monitoring in the perioperative setting. Anesth Analg 2009;108:887-97.

34. Hoit BD. Strain and strain rate echocardiography and coronary artery disease. Circ Cardiovasc Imaging 2011;4:179-90.

35. Ochiai ME, Cardoso JN, Vieira KRN, Lima MV, Brancalhao ECO, Barretto ACP. Predictors of low cardiac output in decompensated severe heart failure. Clinics 2011;66:239-44.

36. Karamanoglu M, Bennett T, Ståhlberg M, Splett V, Kjellström B, Linde C, Braunschweig F. Estimation of cardiac output in patients with congestive heart failure by analysis of right ventricular pressure waveforms. Biomed Eng Online 2011;10:36.

37. Simioni C, Nardozza LMM, Araujo Júnior E, Rolo LC, Zamith M, Caetano AC, Moron AF. Heart stroke volume, cardiac output, and ejection fraction in 265 normal fetus in the second half of gestation assessed by 4D ultrasound using spatio-temporal image correlation. J Matern Fetal Neonatal Med 2011;24:1159-67. 